\title{
Structural Changes of the Liver Vessels in Compensated and Decompensated Coarctation of Aorta in Experimental Studies
}

\author{
S. V. Shormanov, S. V. Kulikov \\ Department of Anatomy, Yaroslavl State Medical University, Yaroslavl, Russia \\ Email: s v shormanov@rambler.ru
}

Received 10 February 2015; accepted 8 March 2015; published 17 March 2015

Copyright @ 2015 by authors and Scientific Research Publishing Inc.

This work is licensed under the Creative Commons Attribution International License (CC BY). http://creativecommons.org/licenses/by/4.0/

c) (i) Open Access

\begin{abstract}
Our studies allowed us to establish that simulating coarctation of aorta in laboratory animals results in tone reduction of vessels carrying blood to the liver and from it and a subsequent development of their intima atrophy. In spite of that fact the number of vessels containing intimal musculature, muscular-elastic sphincters and polypoid pulvini in the arterial bed flow increases and in the major outflowing veins, on the contrary, thinning of their muscular tori takes place. Appearance and functioning in the hepatic vessels of adaptive formations contributes to keeping up homeostasis at the microcirculatory level. With cardiac decompensation decreasing because of venous plethora, hypotonia and atrophy of the intima of the vessels responsible for the blood inflow to the liver and the outflow from it progress. Besides, the number of arteries and the rate of development of arteries with adaptive smooth muscle formations in the arteries, as well as in hepatic veins with muscular tori decreases dramatically. The reason of compensatory reaction failure is a long-term hypoxia leading to vessel and their adaptive formation sclerosis. The consequence of the exhaustion of the above-mentioned mechanisms is development of perisinusoidal fibrosis and depression of the transcapillary metabolism.
\end{abstract}

\section{Keywords}

Coarctation of Aorta, Hepatic Vessels, Compensation, Decompensation

\section{Introduction}

Coarctation of aorta is a common and severe congenital heart disease [1]-[3]. When this pathology occurs there

How to cite this paper: Shormanov, S.V. and Kulikov, S.V. (2015) Structural Changes of the Liver Vessels in Compensated and Decompensated Coarctation of Aorta in Experimental Studies. J. Biomedical Science and Engineering, 8, 123-130. 
appear two hemodynamic basins in the systemic circulation: one lower and the other higher the area of the aorta narrowing [4]. An average life span of such patients without a surgery doesn't exceed 30 years [5]. The progression of the disease and the rate of complications that set in are associated not only with the compensatory potential of the defective heart but also with the degree of structural changes taking place in the liver [6]. The vascular system of the liver has a considerable range of plastic and adaptive resources and due to this fact participates very well in the regulation of the circulatory disorders [7] [8]. It should be noted that in the literature reviews there is scarce information concerning the peculiarities of the structural reconstruction of the hepatic vessels in compensated coarctation of aorta [9]. The data concerning the morphology of the liver is lacking in general. Carrying out the experiments on humans is extremely difficult as the patients differ in age, sex, and have various accompaning diseases. So, the above-mentioned considerations prompted us to conduct an experiment by means of simulating coarctation of aorta in the laboratory animals and using such methods of investigation as morphometry, electronic microscopy and immunohistochemistry.

The aim of this study is to establish the nature of structural changes in the vessels of the liver in experimental coarctation of aorta and define the role of these changes in the development of the mechanisms of compensation and decompensation in both the organ and the systemic circulation.

\section{Material and Methods}

Hemodynamic models of coarctation of aorta have been created in 25 puppies by surgical intervention to achieve this goal in the experiment. For this purpose the narrowing of the aorta isthmus was performed by means of a polyethylene stethoscope and in this way the lumen of the vessel was diminished as much as 55\% [6]. The experiment was conducted in accordance with "The Helsinki Declaration and the Regulations of Carrying out Experiments in Laboratory Animals”. The follow-up period lasted from 6 months to 2 years: there after the animals were sacrificed by phlebotomy under ether narcosis. 9 puppies developed heart failure at different periods of time after the surgery according to the left ventricular type which is characterized by ever increasing pulmonary edema that eventually caused death of these animals. In the control group we used the specimens from 10 puppies of the corresponding age. Samples of the liver were excised from various areas in 3 dimensions, fixed in $10 \%$ neutral formalin and poured into paraffin. Histological sections were stained with matoxylin and eosine according to Masson, Hart and van Gison and also impregnated with silver according to Foot. For electron-microscopic examination the samples of the liver were fixed in glutar aldehyde on phosphate buffer and poured into epoxide resin (mixture of epon and araldit). Monoclonal antibodies to $\alpha$-SMA and CD34, enzymatic avidin-biotin systems of detection and chromogenic substrata (DAB and nikel-DAB) produced by Novocastra (Great Britain) [10] were used for the immunohistochemical reactions. The vascular bed of the blood inflow to the liver (the branches of the hepatic artery and the portal vein) and that of the outflow of blood from the liver were investigated. For that purpose the technique of complex morphometry of the vascular basin of the liver worked out earlier was employed [11]. In accordance with this technique all the hepatic arteries were subdivided into 4 groups: large (will the external diameter of $125 \mu \mathrm{m}$ and more), of medium size (124 - $51 \mu \mathrm{m}$ ), small (50 $21 \mu \mathrm{m})$ and arterioles (20 $\mu \mathrm{m}$ and less). The portal veins depending on the accompanying them arteries were subdivided into 4 levels: The veins corresponding to the level of large arteries (190 $\mu \mathrm{m}$ and more), those corresponding to the level of medium arteries $(189-110 \mu \mathrm{m})$, the veins of the level of small arteries $(109-51 \mu \mathrm{m})$ and finally those of the level of arterioles-venules (50 $\mu \mathrm{m}$ and less). The hepatic veins were also subdivided into 4 types (large, medium, small and venules), the basis for this subdivision being the diameter of the corresponding portal veins. Morphometry of all the vessels that appeared to be caught in the horizontal section was carried out by means of the propeller ocular micrometer of MOB-1-15 $5^{\mathrm{x}}$ type. Along with it the thickness of their medium membrane was measured, the adventitia not being taken into consideration. The number of myocytes in the media of small arteries was also determined. The size of these cells was possible to estimate on the basis of the size of their nuclei [12]. The nucleus area and volume of leuomyocytes were calculated according to the formula: $\mathrm{S}=0.785 \mathrm{~cd}, \mathrm{~V}=0.523 \mathrm{~cd}^{2}$, where $\mathrm{c}$ and $\mathrm{d}$ are length and diameter of the nucleus. Besides, the count of arteries containing intimal musculature, muscular and elastic sphincters and polypoid pulvini was made. At the level of large hepatic veins the thickness of the muscular tori was measured. The digital material was processed by means of STATISTICA program, Student's t-test and with Bonferoni correction for multiple comparisons. The results were considered reliable if the error didn't exceed 5\% ( $<<0.05)$. 


\section{Results and Discussion}

\subsection{Structural Changes in the Hepatic Vessels in Compensated Coarctation of Aorta}

In compensated coarctation of aorta there appear marked structural changes in both the inflowing (branches of the hepatic artery and portal vein) and outflowing (branches of the hepatic vein) vessels of the liver. Abrupt blood pressure lowering in the inflowing branches of the hepatic artery is accompanied by lowering of the tone of its arteries. It is manifested by widening of the internal elastic membrane and weakening of its rugae. Morphometric studies make it possible to establish that the thickness of the intima in large, medium arteries and arterioles dicreases 1.4 times and in small arteries 1.5 times (Table 1). The measurement of the smooth myocite nuclei in the wall of small arteries shows that their length decreases 1.4 times, their diameter-1.5 times, their area and volume-2.1 and 3.2 times, respectively; the number of cells in comparison with the control figures decreases 1.9 times (Table 2). Karyometry data are confirmed by immunoghistochemical examination with determination of $\alpha$-SMA expression. In addition to it in the intima of hepatic arteries lowering of the expression of small muscular actin $\alpha$-SMA takes place, the fact reflecting the decrease of the number of smooth myocytes in comparison with the control data (Figure 1(a) and Figure 1(b)).

At the same time the number of arteries with oblique-longitudinal musculature, muscular-elastic sphincters and polypoid pulvini in the liver increases. Oblique-longitudinal musculature is located in the internal vascular membrane and is represented by both separate cells and their bundles (Figure 2(a)), as well as by the laminae/plates that envelope the vessel lumen all along its perimeter. This testifies to the reconstruction of arteries according to the locked type. At the point of the intimal myocyte bundles location the internal elastic membrane splits into two plates forming a duplicate.

Table 1. Thickness of the medium membrane of the liver (in $\mu \mathrm{m}$ ) in control figures, in compensated and decompensated coarctation of aorta $(\mathrm{M} \pm \mathrm{m})$.

\begin{tabular}{|c|c|c|c|c|c|c|c|c|}
\hline \multirow[t]{3}{*}{ Gr. } & \multicolumn{8}{|c|}{ Basin of blood inflow to the liver } \\
\hline & \multicolumn{4}{|c|}{ Arteries } & \multicolumn{4}{|c|}{ Portal veins of the level of } \\
\hline & Large & Medium & Small & Arterioles & Large arteries & Medium arteries & Small arteries & Arterioles \\
\hline $\mathrm{C}$ & $24.0 \pm 1.1$ & $13.2 \pm 0.8$ & $6.5 \pm 0.1$ & $3.8 \pm 0.09$ & $6.0 \pm 0.2$ & $5.1 \pm 0.3$ & $4.1 \pm 0.2$ & $2.8 \pm 0.1$ \\
\hline $\mathrm{CC}$ & $17.0 \pm 0.9$ & $9.3 \pm 0.3$ & $4.3 \pm 0.1$ & $2.6 \pm 0.05$ & $3.6 \pm 0.1$ & $3.1 \pm 0.1$ & $2.5 \pm 0.1$ & $2.1 \pm 0.1$ \\
\hline $\mathrm{p}_{\mathrm{c}}$ & $<0.001$ & $<0.001$ & $<0.001$ & $<0.001$ & $<0.001$ & $<0.001$ & $<0.001$ & $<0.001$ \\
\hline CD & $16.9 \pm 2.5$ & $8.0 \pm 0.9$ & $3.7 \pm 0.1$ & $2.2 \pm 0.1$ & $3.4 \pm 0.1$ & $2.5 \pm 0.3$ & $2.0 \pm 0.05$ & $1.9 \pm 0.1$ \\
\hline $\mathrm{p}_{\mathrm{c}}$ & $>0.05$ & $>0.05$ & $<0.001$ & $<0.001$ & $<0.001$ & $<0.001$ & $<0.001$ & $<0.001$ \\
\hline $\mathrm{p}_{\mathrm{cc}}$ & $<0.001$ & $<0.001$ & $<0.001$ & $<0.001$ & $>0.05$ & $<0.001$ & $<0.001$ & $>0.05$ \\
\hline \multirow[t]{3}{*}{ Gr. } & \multicolumn{8}{|c|}{ Basin of blood outflow from the liver } \\
\hline & \multicolumn{8}{|c|}{ Hepatic veins } \\
\hline & Large & & Medium & & Small & & Venules & \\
\hline $\mathrm{C}$ & $6.2 \pm 0.3$ & & $4.4 \pm 0.1$ & & $3.2 \pm 0.1$ & & $2.7 \pm 0.1$ & \\
\hline CC & $4.0 \pm 0.2$ & & $3.0 \pm 0.1$ & & $2.4 \pm 0.1$ & & $2.1 \pm 0.1$ & \\
\hline $\mathrm{p}_{\mathrm{c}}$ & $<0.001$ & & $<0.001$ & & $<0.001$ & & $<0.001$ & \\
\hline CD & $3.5 \pm 0.4$ & & $2.5 \pm 0.1$ & & $2.3 \pm 0.08$ & & $2.0 \pm 0.05$ & \\
\hline $\mathrm{p}_{\mathrm{c}}$ & $<0.001$ & & $<0.001$ & & $<0.001$ & & $<0.001$ & \\
\hline $\mathrm{p}_{\mathrm{cc}}$ & $>0.05$ & & $<0.001$ & & $>0.05$ & & $>0.05$ & \\
\hline
\end{tabular}

Note: Gr._-group of experiments; C—control figures; CC—compensated coarctation of aorta; CD—decompensated coarctation of aorta; $\mathrm{P}_{\mathrm{c}}$ — comparison with the control figures; $\mathrm{p}_{\mathrm{cc}}$-comparison with compensated coarctation of aorta. 
Table 2. Parameters of smooth-muscular cells in the medium membrane of small arteries of the liver in control groups, in compensated and decompensated coarctation of aorta $(\mathrm{M} \pm \mathrm{m})$.

\begin{tabular}{|c|c|c|c|c|c|}
\hline Kind of group & $\begin{array}{l}\text { Dlinnik of the } \\
\text { nucleus in } \mu \mathrm{m}\end{array}$ & $\begin{array}{l}\text { Diameter of the } \\
\text { nucleus in } \mu \mathrm{m}\end{array}$ & $\begin{array}{c}\text { Area of the } \\
\text { nucleus in } \mu \mathrm{m}^{2}\end{array}$ & $\begin{array}{l}\text { Volume of the } \\
\text { nucleus in } \mu \mathrm{m}^{3}\end{array}$ & Number of cells \\
\hline C & $7.6 \pm 0.10$ & $4.1 \pm 0.60$ & $24.6 \pm 0.60$ & $72.4 \pm 3.30$ & $9.7 \pm 0.80$ \\
\hline $\mathrm{CC}$ & $5.6 \pm 0.09$ & $2.7 \pm 0.02$ & $11.9 \pm 0.20$ & $22.6 \pm 1.00$ & $5.1 \pm 0.10$ \\
\hline $\mathrm{p}_{\mathrm{c}}$ & $<0.001$ & $<0.001$ & $<0.001$ & $<0.001$ & $<0.001$ \\
\hline CD & $5.5 \pm 0.2$ & $2.7 \pm 0.1$ & $11.7 \pm 0.20$ & $22.0 \pm 1.0$ & $3.7 \pm 0.20$ \\
\hline $\mathrm{p}_{\mathrm{c}}$ & $>0.05$ & $>0.05$ & $>0.05$ & $>0.05$ & $<0.001$ \\
\hline $\mathrm{p}_{\mathrm{cc}}$ & $<0.001$ & $<0.001$ & $<0.001$ & $<0.001$ & $<0.001$ \\
\hline
\end{tabular}

Note: C—control; CC—compensated coarctation of aorta; CD—decompensated coarctation of aorta; $\mathrm{p}_{\mathrm{c}} —$ comparison with the control figures; $\mathrm{p}_{\mathrm{cc}}-$ comparison with compensated coarctation of aorta.

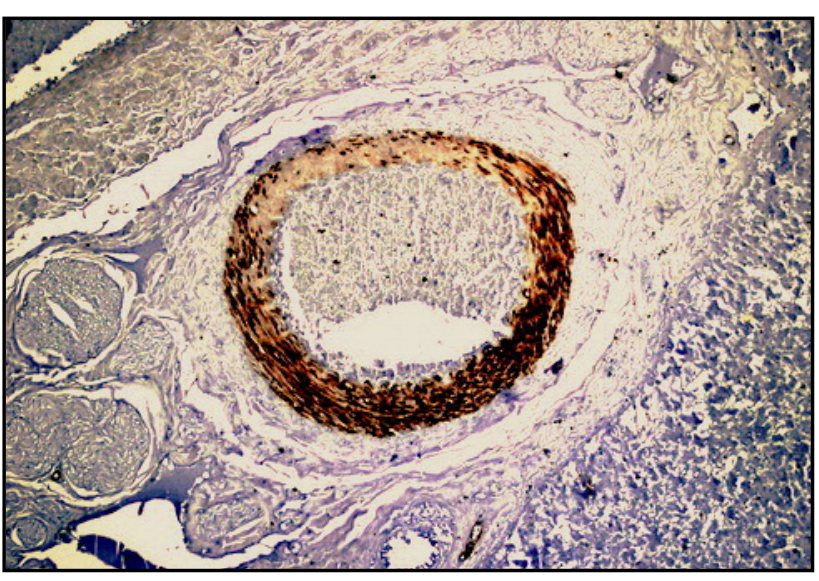

(a)

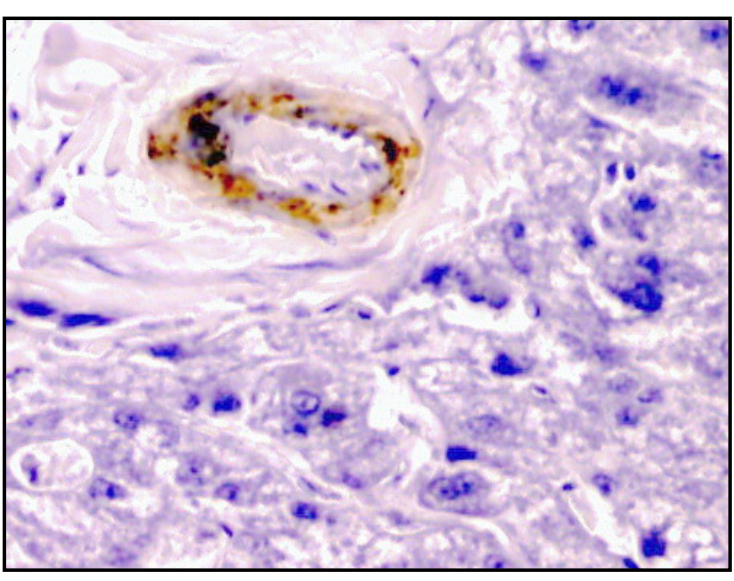

(b)

Figure 1. Immunohistochemical liver vessel changes in control groups (a) and compensated coarctation of aorta (b). (a) high $\alpha$-SMA expression in the medium membrane of the medium artery; (b) low $\alpha$-SMA expression in the medium membrane of the small artery. a-magnification 100; b-magnification 200.

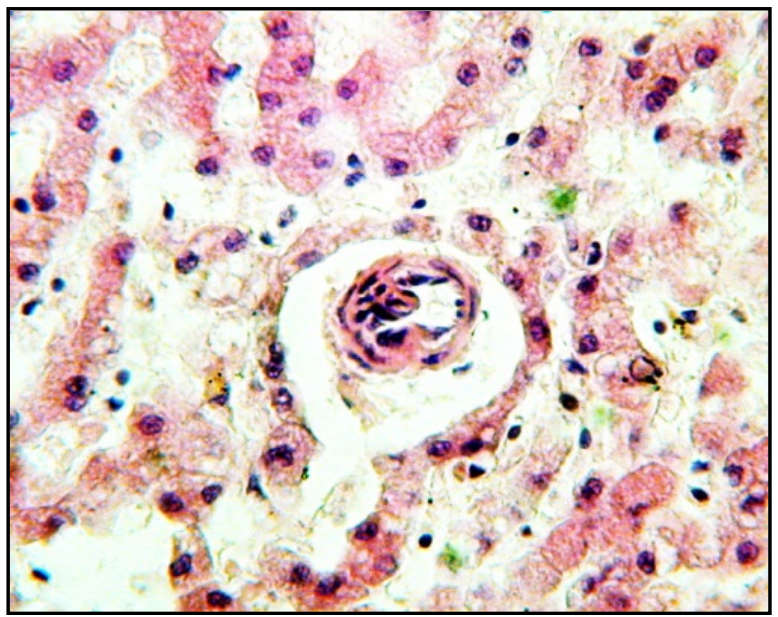

(a)

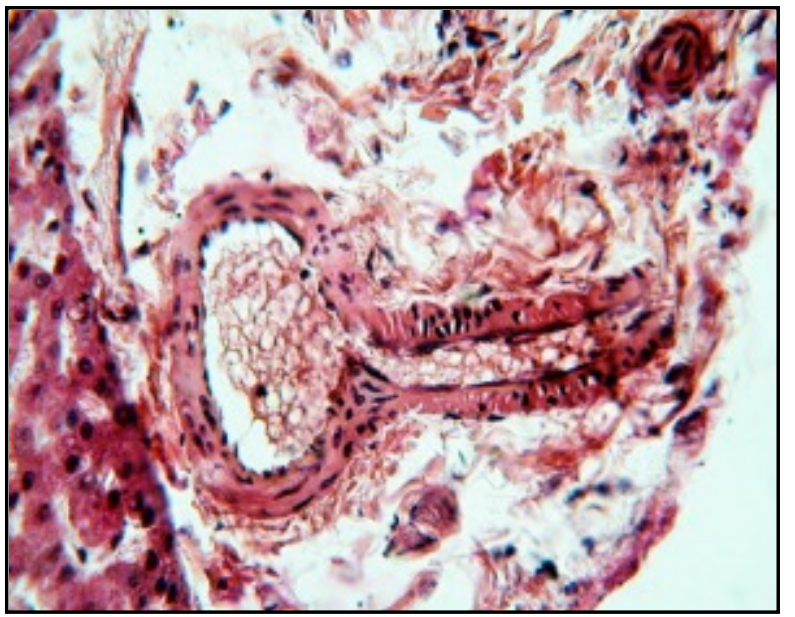

(b)

Figure 2. Structural changes of the hepatic vessels in compensated of coarctation of aorta. (a) Intimal musculature bundles in a small artery; (b) Muscular-elastic sphincter in the orifice of a medium artery. a, b-magnification 200. Hematoxylin and eosine staining. 
Our calculations show that among large arteries and arterioles the number of vessels with oblique-longitudinal smooth muscles increases 3-fold, and at the level of medium and small arteries-1.5 and 18 times, respectively. Muscular-elastic sphincters represent formations that surround the ostia of lateral branches of arteries all along the perimeter and at the longitudinal section of such a branch they look like one torus or two tori coming out into the lumen of the main vessel (Figure 2(b)) on the horizontal section. The above-mentioned torus or tori consist of a mass of leomyocytes lying between two plates of the inner elastic membrane. The number of vessels with sphincters in the area where large arteries and arteriols branch increases 2-fold, at the level of medium arteries2.5 times and at the level of small arteries-4-fold. Polypoid pulvini of the arteries have a round or an oval shape and a pedicle connecting the above mentioned formations with the wall of the vessel. In such cases they look like a polyp. Depending on their histological structure we distinguish 3 types of pulvini. The first type pulvini consists of a subintimal layer protruding into the lumen and surrounded by endothelium. The second type pulvini consists of an invaginated part containing intertwining bundles of smooth musculature making their way in different directions. The peripheral part of the third type pulvini is made up of smooth muscular tissue and the central one of connective tissue formed by fibrous adventitia structures and fibroblasts. Such formations are called Conti pulvini in morphological literature [13]. In experiment studies they are most frequently to be found at the level of medium and large arteries but in control groups they are not to be found at all.

Considerable structural changes in coarctation of aorta take place not only in the arteries of the liver but also in the branches of the portal vein located nearby. Elastic membrane winding of these vessels becomes less marked and testifies to their tone lowering. The portal vein wall noticeably thins out regardless of their caliber. In the veins of the large, medium and small arteries level it decreases 1.6 times, and in the veins of arteriole level—1.3 times (Table 1 ).

In the sinusoids of the liver histological and electromicroscopical changes appear to be minimal. The vessels seem to be a little dilated but structurally they hardly differ from those in the control group. Immunohistological examination doesn't reveal CD34 expression in the sinusoids, the fact indicating the integrity of their basal phenystril membrane.

In the vessels belonging to the vascular bed system responsible for the blood outflow from the liver (hepatic veins) we observed marked structural changes. Their large and medium branches wall thickness decreased 1.5 times and that of small veins and venules-1.3 times (Table 1). Muscular tori of the hepatic veins play a significant role in the blood outflow from the liver in dogs. In medical literature the vessels containing such muscular tori got the name of Mautner-Pick veins [6]. Histologically at the longitudinal section these veins have a serrate inner contour because the bundles of the smooth musculature jut out into the lumen. If the section is made horizontally through these bundles the protrusion of these formations into the vein lumen is to be seen very well and not infrequently they even resemble arteries according to the extent of development of their muscular membrane. The stroma of the muscular tori is represented by a powerful argyrophyl shell and collagen fibers. As our experiments have demonstrated these tori in simulated coarctation of aorta grow thin. Actually their thickness on average decreases 1.8 times $(\mathrm{p}<0.001)$.

\subsection{Structural Changes of Liver Vessels Due to Decompensated Coarctation of the Aorta}

In the case of decompensated coarctation of aorta morphological changes in the vascular system of the liver are more marked. The inner elastic membrane of the arteries not only becomes smooth, but unevenly wears out as well, testifying a high degree of hypotension. Atrophic changes of middle coat of the liver arteries occur to be more pronounced than in the previous series of experiments, which quantitatively proves morphometry findings. In addition, wall thickness of arterioles and small arteries decreases 1.7 times, medium ones-1.6 times, and large ones-1.4 times (see Table 1). Karyometry findings have determined linear and calculated ratings of smooth myocyte nuclei decrease in the same proportions as in the previous experimental series. However the amount of cells decrease is greater-2.6 times (see Table 2). Immunohistochemical research shows a pronounced reduction of smooth muscle actin $\alpha$-SMA in arterial walls in comparison with compensated coarctation. That is shown in progressing decrease in the number of smooth myocytes.

Apart from the enumerated facts, this experimental series has shown arteries reconstructed according to locked type more rarely. For example, the amount of vessels (arterioles and small arteries) with intimal muscular structure decreases 3 and 9 times compared to compensated coarctation. Muscular-elastic sphincters show sclerosis and hyalinosis. These structures amount arterioles and small arteries, compared to the animals in the $1^{\text {st }}$ 
observation group, becomes 1.5 and 4 times less. Polypoid formations in liver bloodstream never occur in the series of dogs. Connective tissue growth is marked against the background of smooth muscles atrophy in many arteries middle coat.

There are not only changes in rugosity in the branches of the portal vein, but also pronounced wearing out of elastic plates. The middle coat thickness of these vessels decreases in the veins compared to small and medium arteries level 2 times, in the veins compared to arterioles level-1.5 times, in the veins compared to large arteries level-1.8 times.

The sinusoids of liver lobes appear to be extremely plethoric. Electronograpms show thickening of their basal membranes, collagen deposition in dilated perisinusoidal space (perisinusoidal fibrosis), causing continuous basal membrane formation, similar to that of capillaries in other organs. The electronographic microscopy findings are proved by immunohistochemical research, defining the expression of CD34. In decompensated coarctation of aorta, compared to the compensated one, and as a result of sclerosis, continuous basic membrane formation and perisinusoidal fibrosis, endothelium actively expresses CD34 (Figure 3(a) and Figure 3(b)).

Marked plephora is noted in the bloodstream from the liver, as opposed to the dogs with compensated coarctation. Media thickness in venules and small liver veins decreases 1.4 times, in medium and large ones-1.8 times (Table 1). At the same time even greater atrophy of muscular cylinders is noted in Mautner-Pick veins and also these veins' sclerosis. The average thickness of cylinders decreases 2 times $(\mathrm{p}<0.001)$.

\section{Conclusion}

Thus, the research has shown that coarctation of aorta simulated in the experiment is followed by the formation of pronounced structural changes in the vascular system of the liver. In compensated coarctation of aorta the blood inflow to this organ under lowered pressure leads to hypotension and dilatation of the inflowing vessels. Due to decreased hemodynamic load the walls of arteries and portal veins of different size wear out and undergo atrophy. The phenomena are based on their media leiomyocytes decrease in number and size, which can be considered an adjustment. As a result of new conditions of blood circulation the amount of arteries, containing bundles of oblique and longitudinal smooth myocytes, muscular-elastic sphincters and polypoid formations is increasing. According to our research and literature findings such formations sometimes occur in the bloodstream of control animals [6] [11] [14]. These formations are based on medium membrane of the liver arteries myocytes migrating into the intima through "openings" in the inner elastic membrane [6] [11]. By contracting and dilating these muscular structures regulate the arterial blood supply of the liver providing the optimal supply of its parenchima in chronic ischaemia, and subsequently serve as adjustment formations. As a response to lowering of blood pressure in the vessels carrying blood to the liver there develops hypotonia in the vessels carrying blood from the liver. This results in wearing out of the liver veins walls and muscular tori in Mautner-Pick veins playing an important role in blood depositions in dogs [7] [11]. This also leads to retaining blood in the liver veins

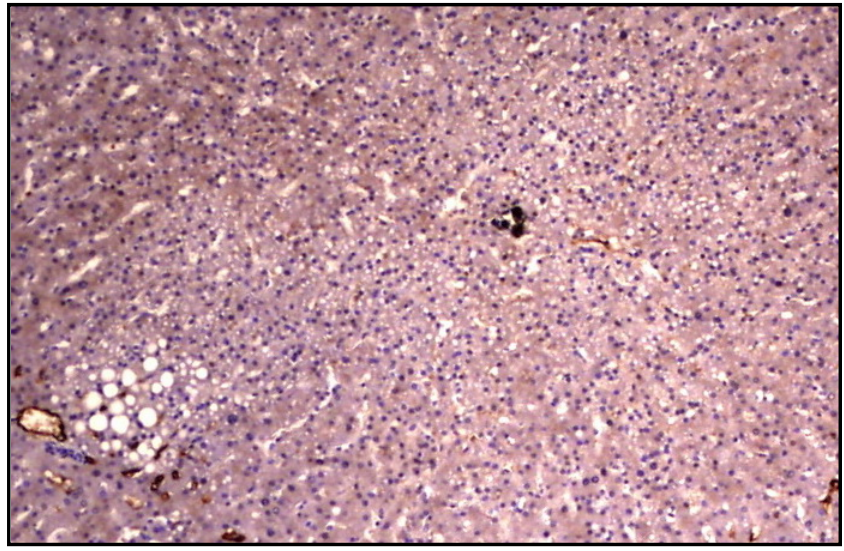

(a)

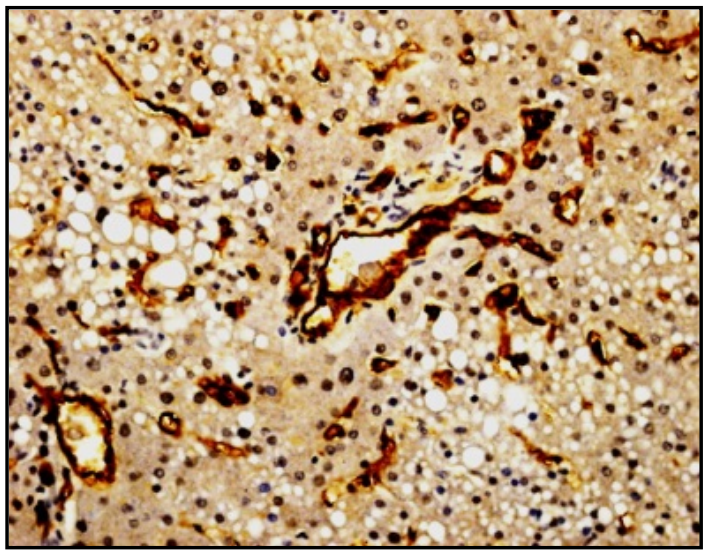

(b)

Figure 3. Immunohistochemical changes of liver vessels due to compensated (a) and decompensated (b) coarctation of aorta. (a) Lack of expression CD34 in the sinusoids; (b) High expression in the sinusoids. a-magnification 100; b-magnification 200. 
on contraction of the muscular tori and its discharge into the systemic circulation on their relaxation. In our opinion, depositing function of the liver in coarctation of aorta is decreased as otherwise its proper functioning would lead to the decrease of the blood supply in the arterial systemic circulation, where persistent hypotension has set in. Morphologically this decrease means atrophy of muscular tori. Thus, the function of the adjustment structures, situated on different sides of the sinusoids (bundles of intimal musculature, muscular sphincters and polypoid pulvini — on one side and muscular tori of the veins — on the other side) is essentially different. In afferent vessels they regulate the flow of blood on the organ (liver) level, in the vessels carrying blood from the liver they regulate the volume of blood in systemic circulation.

According to our research, structural changes in the hepatic vessels are considerably different in compensated and decompensated defect. This is directly associated with the differences in the degree of blood supply disorders. Decompensated coarctation of aorta is manifested by the left ventricular cardiac insufficiency which causes congestion of blood in pulmonary circulation and pulmonary edema of hemodynamic genesis. Insufficiency of the left chambers of the heart is accompanied by contractile function impairment of the right chamber as well; that is why all the animals at the stage of decompensation demonstrated venous plephora. Venous haemostasia causes dramatic dilatation of veins carrying blood from the liver and sinusoids. Tissue metabolism dysfunction at the sinusoid level creates the increase of hypoxia, the fact that causes even greater than in compensated coarctation, tone lowering of the afferent vessels and more pronounced atrophic changes in their medium membrane. Animals with decompensated defect show substantial structural changes of afferent liver vessels, thus morphologically reflecting sinusoidal homeostasis failure. These are to be observed in the fact that the number of arteries with intimal musculature, muscular-elastic sphincters and polypoid pulvini is considerably less in this series of experiments than in the animals of the first one. Adjustment structures show sclerotic changes, causing their functional failure, that is inability to regulate the impaired hemodynamics of the organ. Besides, smooth muscles sclerotic formations not only fail to regulate the organ circulation but also form a mechanical obstacle in the arterial bloodstream, jutting out into the lumen of the arteries. The same reaction is to be found in the veins carrying blood from the liver where atrophy and sclerosis of the muscular tori of Mautner-Pick veins alongside with the lowering of their contractile properties takes place, the fact contributing to the venous congestion development. Enumerated changes reflect the exhaustion of compensatory reactions of the liver vessels and their inability to protect the microcirculatory system from blood overflow. In the latter there develops perisinusoidal fibrosis, thickening and formation of sinusoids continuous basal membranes, which causes transcapillary exchange decrease, aggravating hypoxia and creating a vicious circle. Obviously, adjustment insufficiency is an unavoidable consequence of a higher level sclerotic changes in the liver vessels and their regulating structures in decompensated coarctation. All these facts serve as a basis for the forthcoming liver dysfunction.

\section{Findings}

1) Simulating coarctation of aorta in experimental animals is accompanied by the liver circulatory impairment that is revealed in the decrease of the blood supply to this organ with the subsequent development of a complex of adjustment changes in the vessels as well as that of pathological changes.

2) Adjustment changes in the vessels carrying blood to the liver and from it in the compensated stage are seen in vasodepression, decrease of the degree of smooth muscles development, as well as in greater intensity of development of adjustment structures regulating the blood supply in the microcirculatory system.

3) The transition of compensated defect to decompensated one is not only associated with even greater vasodepression, the vessels medium membrane atrophy but also with a considerable involution of adjustment structures that have been formed before. In decompensated coarctation of aorta atrophy of smooth muscles is accompanied by such pathological changes as excrescence of rough fibrous connective tissue. Sclerosis affects not only arterial and venous walls but their adjustment formations as well, the fact transforming the latter into hardlycontractible structures preventing the flow of blood.

4) The development of the liver hemocirculatory disorders, along with the development of cardiac decompensation and sclerosis of vessels, results in sinusoids capillarization, negatively affecting the organ functioning.

\section{References}

[1] Bankl, G. (1980) Congenital Heart and Large Vessels. Moscow.

[2] Belokon, N.A. and Podzolkov, V.P. (1991) Congenital Heart Disease. Medicine, Moscow. 
[3] Rao, P.S. (1995) Coarctation of the Aorta. J. Semin-Nephrol., 15, 50-87.

[4] Sharykin, A.S. (2005) Congenital Heart Disease. Medicine, Moscow.

[5] Mutaffyan, O.A. (2002) Congenital Heart Disease in Children. Neva Dialect, Petersburg.

[6] Shormanov, S.V. and Kulikov, S.V. (2003) Morphological Changes in Blood Vessels of the Liver in Experimental Coarctation of the Aorta and after Its Removal. Morphology, 124, 61-66.

[7] Esipova, I.K., Kaufman, O.Y. and Kryuchkova, G.S. (1971) Essays on Hemodynamic Restructuring Vascular Wall. Medicine, Moscow.

[8] Kapustina, E.V. (1977) Reaction Vessels Leading to the Liver Experimental Venous Stasis in the Early Stages of the Experiment. Archives of Anatomy, Histology and Embryology, 12, 68-72.

[9] Kreuser, W., Sykes, B. and Schenk, W. (1973) Hepatic Hemodynamics in Experimental Coarctation of the Aorta. Vasa, 2, 51-57.

[10] Kiyasov, A.P., Odintsov, A.H., Gumerova, A.A. and Gazizov, I.M. (2008) Autologous Transplantation of Hematopoietic Stem Cells to Patients with Chronic Hepatitis. Cell Transplantation and Tissue Engineering, 3, 70-75.

[11] Shormanov, S.V. and Kulikov, S.V. (2001) Structural Changes in the Liver Arteries Intraorganic Coarctation of the Aorta in the Experiment. Morphology, 122, 25-29.

[12] Kaufman, O.Y. (1979) Hypertrophy and Smooth Muscle Regeneration. Science, Moscow.

[13] Conti, G. (1958) Sur La morphologie des anastomoses arterioveinnses et des dispositits regulateurs du courant sanguine. J. Ann. Anat. Pathol., 1, 5-32.

[14] Shormanov, S.V. and Yaltsev, A.V. (1996) State of the Arterial System of the Kidneys in Experimental Coarctation of the Aorta and after Its Removal. Archives of Pathology, 58, 37-41. 